\title{
Numerical Solutions to Neutral Stochastic Delay Differential Equations with Poisson Jumps under Local Lipschitz Condition
}

\author{
Jianguo Tan, ${ }^{1}$ Hongli Wang, ${ }^{2}$ Yongfeng Guo, ${ }^{1}$ and $\mathrm{Zhiwen} \mathrm{Zhu}^{2}$ \\ ${ }^{1}$ Department of Mathematics, Tianjin Polytechnic University, Tianjin 300387, China \\ ${ }^{2}$ Department of Mechanics, Tianjin University, Tianjin 300072, China \\ Correspondence should be addressed to Jianguo Tan; tanjg@tju.edu.cn
}

Received 13 February 2014; Revised 18 May 2014; Accepted 19 May 2014; Published 4 June 2014

Academic Editor: Mohamed Abd El Aziz

Copyright (c) 2014 Jianguo Tan et al. This is an open access article distributed under the Creative Commons Attribution License, which permits unrestricted use, distribution, and reproduction in any medium, provided the original work is properly cited.

\begin{abstract}
Recently, Liu et al. (2011) studied the stability of a class of neutral stochastic delay differential equations with Poisson jumps (NSDDEwPJs) by fixed points theory. To the best of our knowledge to date, there are not any numerical methods that have been established for NSDDEwPJs yet. In this paper, we will develop the Euler-Maruyama method for NSDDEwPJs, and the main aim is to prove the convergence of the numerical method. It is proved that the proposed method is convergent with strong order $1 / 2$ under the local Lipschitz condition. Finally, some numerical examples are simulated to verify the results obtained from theory.
\end{abstract}

\section{Introduction}

Neutral stochastic delay differential equations (NSDDEs) have recently been studied intensively by, for instance, Kolmanovskii et al. [1, 2], Mao et al. [3-6], Luo et al. [7], Zhou and $\mathrm{Hu}$ [8], and Luo [9]. However, explicit solutions can hardly be obtained for NSDDEs; as a result, several numerical schemes have been developed to produce approximate solutions for NSDDEs. For example, Wu and Mao [10] studied the numerical solutions of NSDDEs. Zhang and Gan [11] considered the mean square convergence of one-step methods for NSDDEs. Zhou and Wu [12] studied the convergence of numerical solutions to neutral stochastic delay differential equations with Markovian switching. Poisson jumps are becoming increasingly used to model real-world phenomena in different fields such as economics, finance, biology, and physics. There is an extensive literature concerned with Poisson jumps. For example, Wang et al. $[13,14]$ studied the semi-implicit Euler method for stochastic differential delay equation with jumps (SDDEwJs) and the convergence of numerical solutions to stochastic differential delay equations with Poisson jump and Markovian switching (SDDEwPJMSs). Li et al. $[15,16]$ discussed the convergence of the numerical solutions for SDDEwJs and SDDEwPJMSs. Luo [17] considered the comparison principle and stability of SDDEwPJMSs. Therefore it is natural and necessary to incorporate jumps in the neutral stochastic delay differential equations. However, the study of neutral stochastic delay differential equations with Poisson jumps is less by far. Cen and Zhou [18] investigated convergence of numerical solutions to neutral stochastic delay differential equation with Poisson jump and Markovian switching. Liu et al. [19] studied the stability of NSDDEwPJs by using fixed points theory. Luo and Taniguchi [20] proved the existence and uniqueness for non-lipschitz stochastic neutral delay evolution equations driven by Poisson jumps. However, there are not any numerical methods that have been established for NSDDEwPJs yet. Therefore, in this paper, we first prove the Euler-Maruyama method applied to NSDDEwPJs converges to the true solution under local Lipschitz condition.

The outline of the paper is as follows. In Section 2 we will introduce some necessary notations and assumptions, and then the Euler-Maruyama method is used to define the numerical solutions for NSDDEwPJs. Section 3 will present several useful lemmas. In Section 4, we state our main result; that is, the numerical solutions will converge to the true solutions of NSDDEwPJs under the local Lipschitz condition. At last, some numerical examples are given to verify the results obtained from the theory. 


\section{Preliminaries and the Euler-Maruyama Approximation}

Let $(\Omega, \mathscr{F}, P)$ be a complete probability space with filtration $\left\{\mathscr{F}_{t}\right\}_{t \geq 0}$, which satisfies the usual conditions, that is, the filtration is continuous on the right and $\mathscr{F}_{0}$ contains all $P$ null sets. Let $C\left([a, b] ; R^{n}\right)$ denote the family of functions $\varphi$ from $[a, b]$ to $R^{n}$ that are right-continuous and have limits on the left; $C\left([a, b] ; R^{n}\right)$ is equipped with the norm $\|\phi\|=$ $\sup _{a<t<b}|\phi(t)|$, where $|\cdot|$ is the Euclidean norm in $R^{n}$, that is, $|x|=\sqrt{x^{T} x}\left(x \in R^{n}\right)$. If $A$ is a vector or matrix, its trace norm is denoted by $|A|=\sqrt{\operatorname{trace}\left(A^{T} A\right)}$, while its operator norm is denoted by $\|A\|=\sup \{|A x|:|x|=1\}$. Let $p>0$ denote by $L_{\mathscr{F}_{0}}^{p}\left([-\tau, 0] ; R^{n}\right)$ the family of all $\mathscr{F}_{0}$ measurable, $C\left([a, b] ; R^{n}\right)$-valued random variables $\xi$ such that $E\|\xi\|^{p}<\infty$. For simplicity, we also denote by $a \wedge b=\min \{a, b\}$ that $a \vee b=\max \{a, b\}$.

In this paper, we consider the $n$-dimensional neutral stochastic delay differential equations with Poisson jumps

$$
\begin{aligned}
& \mathrm{d}[x(t)-G(x(t-\tau))] \\
& =f(x(t), x(t-\tau)) \mathrm{d} t+g(x(t), x(t-\tau)) \mathrm{d} W(t) \\
& +h(x(t), x(t-\tau)) \mathrm{d} N(t), \quad t \in[0, T], \\
& x(t)=\xi(t), \quad t \in[-\tau, 0],
\end{aligned}
$$

where $\tau$ is a positive fixed delay, and $\xi(t) \in L_{\mathscr{F}_{0}}^{p}\left([-\tau, 0] ; R^{n}\right)$, $W(t)$ is a $d$-dimensional standard Wiener process which is $\mathscr{F}_{t}$-adapted, and $N(t)$ is a scalar Poisson process with intensity $\lambda$. Assume that $W(t)$ and $N(t)$ are independent of $\mathscr{F}_{0}$. Moreover the functions $G: R^{n} \rightarrow R^{n}, f: R^{n} \times R^{n} \rightarrow R^{n}$, $g: R^{n} \times R^{n} \rightarrow R^{n \times d}, h: R^{n} \times R^{n} \rightarrow R^{n}$.

The stochastic integral is defined in the Itô sense, and the integral version of (1) is frequently expressed as

$$
\begin{aligned}
x(t) & -G(x(t-\tau)) \\
= & \xi(0)-G(x(-\tau))+\int_{0}^{t} f(x(s), x(s-\tau)) \mathrm{d} s \\
& +\int_{0}^{t} g(x(s), x(s-\tau)) \mathrm{d} W(s) \\
& +\int_{0}^{t} h(x(s), x(s-\tau)) \mathrm{d} N(s) .
\end{aligned}
$$

We can now define the Euler-Maruyama approximate solution for NSDDEwPJs.

Given a step-size $\Delta \in(0,1)$ which satisfies $\Delta=\tau / m$ for an integer $m$, let $t_{k}=k \Delta$ for $k>0$. Compute the discrete approximation $y_{k} \approx x\left(t_{k}\right)$ by setting $y_{0}=x\left(t_{0}\right)$ and performing

$$
\begin{aligned}
y_{k+1} & -G\left(y_{k+1-m}\right) \\
= & y_{k}-G\left(y_{k-m}\right)+f\left(y_{k}, y_{k-m}\right) \Delta \\
& +g\left(y_{k}, y_{k-m}\right) \Delta W_{k}+h\left(y_{k}, y_{k-m}\right) \Delta N_{k} ;
\end{aligned}
$$

if $t_{k}<0$, we have $y_{k}=\xi\left(t_{k}\right)$. Moreover, the increments $\Delta W_{k}:=W\left(t_{k+1}\right)-W\left(t_{k}\right)$ are independent Gaussian random variables with mean 0 and variance $\Delta ; \Delta N_{k}:=N\left(t_{k+1}\right)-N\left(t_{k}\right)$ are independent Poisson distributed random variables with mean $\lambda \Delta$ and variance $\lambda \Delta$.

Let $\tilde{y}(t)=y_{k}, \tilde{y}(t-\tau)=y_{k-m}, t \in\left[t_{k}, t_{k+1}\right)$, with initial values $\tilde{y}(t)=\xi(t)$ on $[-\tau, 0]$. The continuous EulerMaruyama approximate solution $y(t)$ is to be interpreted as the stochastic integral

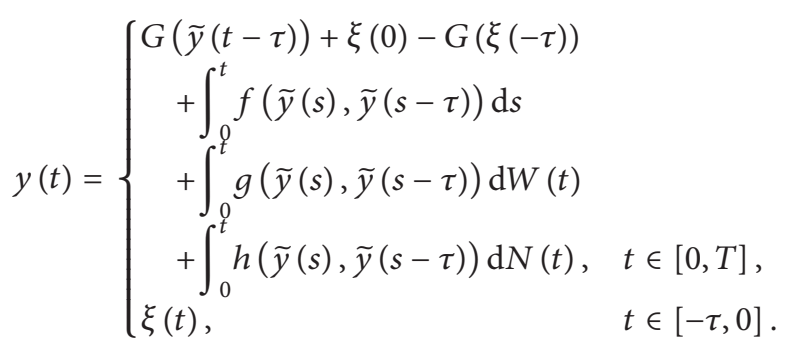

It is not difficult to see that $y\left(t_{k}\right)=\tilde{y}\left(t_{k}\right)=y_{k}$; that is, $y(t)$ and $\tilde{y}(t)$ coincide with the discrete solution at the grid points.

In this paper, the following hypotheses are imposed on (1)

(H1) The Local Lipschitz Condition. There is a positive constant $C_{d}$ such that, for all $x_{1}, x_{2}, y_{1}, y_{2} \in R^{n}$ with $\left|x_{1}\right| \vee\left|x_{2}\right| \vee$ $\left|y_{1}\right| \vee\left|y_{2}\right| \leq d$,

$$
\begin{aligned}
& \left|f\left(x_{1}, y_{1}\right)-f\left(x_{2}, y_{2}\right)\right|^{2} \vee\left|g\left(x_{1}, y_{1}\right)-g\left(x_{2}, y_{2}\right)\right|^{2} \\
& \quad \vee\left|h\left(x_{1}, y_{1}\right)-h\left(x_{2}, y_{2}\right)\right|^{2} \\
& \leq C_{d}\left(\left|x_{1}-x_{2}\right|^{2}+\left|y_{1}-y_{2}\right|^{2}\right) .
\end{aligned}
$$

(H2) The Constractive Mapping. There is a positive constant $K \in(0,1)$ such that, for all $x, y \in R^{n}$

$$
|G(x)-G(y)|<K|x-y|
$$

(H3) The Linear Growth Condition. There is a $L>0$ such that for all $x, y \in R^{n}$

$$
|f(x, y)|^{2}+|g(x, y)|^{2}+|h(x, y)|^{2} \leq L\left(1+\left|x^{2}\right|+\left|y^{2}\right|\right) .
$$

Remark 1. Condition (H3) can be replaced by condition (H1).

In fact, from (5) and inequality $\left|a^{2}+b^{2}\right| \leq 2\left(\left|a^{2}\right|+\left|b^{2}\right|\right)$, it is easy to obtain

$$
\begin{aligned}
|f(x, y)|^{2} & \leq 2|f(x, y)-f(0,0)|^{2}+2|f(0,0)|^{2} \\
& \leq 2 C_{d}\left(|x|^{2}+|y|^{2}\right)+2|f(0,0)|^{2} \\
& \leq L_{d}\left(1+|x|^{2}+|y|^{2}\right)
\end{aligned}
$$


similarly,

$$
\begin{aligned}
& |g(x, y)|^{2} \leq L_{d}\left(1+|x|^{2}+|y|^{2}\right), \\
& |h(x, y)|^{2} \leq L_{d}\left(1+|x|^{2}+|y|^{2}\right),
\end{aligned}
$$

where

$$
L_{d}=2\left(C_{d} \vee|f(0,0)|^{2} \vee|g(0,0)|^{2} \vee|h(0,0)|^{2}\right) .
$$

From (6), we obtain

$$
\begin{aligned}
|G(x)|^{2} & \leq 2|G(x)-G(0)|^{2}+2|G(0)|^{2} \\
& \leq 2 K^{2}|x|^{2}+2|G(0)|^{2} \\
& \leq K_{1}\left(1+|x|^{2}\right),
\end{aligned}
$$

where $K_{1}=2\left(K^{2} \vee|G(0)|^{2}\right)$.

Recently we have studied the existence and uniqueness of solutions to neutral stochastic functional differential equations with Poisson jumps [21]. In an analogous way, we may establish the following existence and uniqueness conclusion that under assumptions (H1)-(H2), (1) has a unique solution $x(t)$ on $t \geq-\tau$.

\section{Lemmas and Corollaries}

In this section, we first establish a few lemmas under local Lipschitz condition. For each $d>0$, define the stopping times

$$
\begin{gathered}
\sigma_{d}:=\inf \{t \geq 0,|y(t)| \geq d\}, \\
v_{d}:=\inf \{t \geq 0,|x(t)| \geq d\}, \\
\rho_{d}=v_{d} \wedge \sigma_{d} .
\end{gathered}
$$

(As usual we set $\inf \emptyset=\infty$.)

Lemma 2. Under (H1)-(H2), for any $p \geq 2$, there exists a constant $A>0$ independent of $\Delta$, such that

$$
E\left(\sup _{-\tau \leq t \leq T}|x(t)|^{p}\right) \vee E\left(\sup _{-\tau \leq t \leq T}|y(t)|^{p}\right)<A .
$$

Proof. First, we prove the $p$-moment of the exact solution of (1) is finite. For any $t_{1} \in[0, T]$, we obtain

$$
E\left(\sup _{-\tau \leq t \leq t_{1}}|x(t)|^{p}\right) \leq E\|\xi\|^{p}+E\left[\sup _{0 \leq t \leq t_{1}}|x(t)|^{p}\right] .
$$

From (2) and inequality

$$
\begin{aligned}
& (a+b+c+d+e+f)^{p} \\
& \quad \leq 6^{p-1}\left(|a|^{p}+|b|^{p}+|c|^{p}+|d|^{p}+|e|^{p}+|f|^{p}\right),
\end{aligned}
$$

we get

$$
\begin{aligned}
& E\left[\sup _{0 \leq t \leq t_{1}}|x(t)|^{p}\right] \\
& \leq 6^{p-1} E\left[\sup _{0 \leq t \leq t_{1}}|G(x(t-\tau))|^{p}\right] \\
& \quad+6^{p-1} E|\xi(0)|^{p}+6^{p-1} E|G(\xi(-\tau))|^{p}
\end{aligned}
$$

$$
\begin{aligned}
& +6^{p-1} E\left[\sup _{0 \leq t \leq t_{1}}\left|\int_{0}^{t} f(x(s), x(s-\tau)) \mathrm{d} s\right|^{p}\right] \\
& +6^{p-1} E\left[\sup _{0 \leq t \leq t_{1}}\left|\int_{0}^{t} g(x(s), x(s-\tau)) \mathrm{d} W(s)\right|^{p}\right] \\
& +6^{p-1} E\left[\sup _{0 \leq t \leq t_{1}}\left|\int_{0}^{t} h(x(s), x(s-\tau)) \mathrm{d} N(s)\right|^{p}\right] .
\end{aligned}
$$

By condition (H2) and inequality $(a+b)^{p} \leq 2^{p-1}\left(|a|^{p}+|b|^{p}\right)$, then for any $t \in[0, T]$, we have

$$
\begin{aligned}
\mid G & \left.(x(t-\tau))\right|^{p} \\
& \leq 2^{p-1}|G(x(t-\tau))-G(0)|^{p}+2^{p-1}|G(0)|^{p} \\
& \leq 2^{p-1} K^{p}|x(t-\tau)|^{p}+2^{p-1}|G(0)|^{p} \\
& \leq L_{p}\left(1+|x(t-\tau)|^{p}\right),
\end{aligned}
$$

where $L_{p}=2^{p-1}\left(K^{p} \vee|G(0)|^{p}\right)$. Thus for any $t_{1} \in[0, T]$,

$$
\begin{aligned}
& E\left[\sup _{0 \leq t \leq t_{1}}|G(x(t-\tau))|^{p}\right] \\
& \quad \leq L_{p}\left(1+E\left[\sup _{0 \leq t \leq t_{1}}|x(t-\tau)|^{p}\right]\right) \\
& \quad \leq L_{p}\left(1+E\left[\sup _{0 \leq t \leq T}|x(t-\tau)|^{p}\right]\right) .
\end{aligned}
$$

Similarly

$$
E\left[\sup _{0 \leq t \leq t_{1}}|G(\xi(-\tau))|^{p}\right] \leq L_{p}\left(1+E\|\xi\|^{p}\right) .
$$

By the Hölder inequality and (8), then for any $t_{1} \in[0, T]$, we get

$$
\begin{aligned}
& E\left[\sup _{0 \leq t \leq t_{1}}\left|\int_{0}^{t} f(x(s), x(s-\tau)) \mathrm{d} s\right|^{p}\right] \\
& \quad \leq T^{p-1} \int_{0}^{t} E|f(x(s), x(s-\tau))|^{p} \mathrm{~d} s \\
& \quad \leq T^{p-1} L_{d}^{p / 2} \int_{0}^{t}\left(1+E|x(s)|^{2}+E|x(s-\tau)|^{2}\right)^{p / 2} \mathrm{~d} s \\
& \quad \leq T^{p-1} L_{d}^{p / 2} \int_{0}^{t}\left(1+2 E \sup _{-\tau \leq \nu \leq s}|x(\nu)|^{2}\right)^{p / 2} \mathrm{~d} s \\
& \quad \leq T^{p-1} L_{d}^{p / 2} 2^{p / 2} \int_{0}^{t}\left(1+E \sup _{-\tau \leq \nu \leq s}|x(v)|^{2}\right)^{p / 2} \mathrm{~d} s \\
& \leq T^{p-1}\left(2 L_{d}\right)^{p / 2}\left(T+\int_{0}^{t_{1}} E \sup _{-\tau \leq \nu \leq s}|x(\nu)|^{p} \mathrm{~d} s\right) .
\end{aligned}
$$


Now, we use $H$ to denote that a generic constant may change between occurrences. Using the Burkholder-Davis-Gundy inequality for the two martingale integral terms, we have

$$
\begin{aligned}
& E\left[\sup _{0 \leq t \leq t_{1}}\left|\int_{0}^{t} g(x(s), x(s-\tau)) \mathrm{d} W(s)\right|^{p}\right] \\
& \leq H \int_{0}^{t_{1}} E|g(x(s), x(s-\tau))|^{p} \mathrm{~d} s \\
& \leq\left(2 L_{d}\right)^{p / 2} H\left(T+\int_{0}^{t_{1}} E \sup _{-\tau \leq \nu \leq s}|x(\nu)|^{p} \mathrm{~d} s\right) .
\end{aligned}
$$

Similarly, for the jump integral term, we have

$$
\begin{aligned}
& E\left[\sup _{0 \leq t \leq t_{1}}\left|\int_{0}^{t} h(x(s), x(s-\tau)) \mathrm{d} N(s)\right|^{p}\right] \\
& =E\left[\sup _{0 \leq t \leq t_{1}} \mid \int_{0}^{t} h(x(s), x(s-\tau)) \mathrm{d} \widetilde{N}(s)\right. \\
& \left.\quad+\left.\lambda \int_{0}^{t} h(x(s), x(s-\tau)) \mathrm{d} s\right|^{p}\right] \\
& \leq 2^{p-1} E\left[\sup _{0 \leq t \leq t_{1}}\left|\int_{0}^{t} h(x(s), x(s-\tau)) \mathrm{d} \widetilde{N}(s)\right|^{p}\right] \\
& \quad+2^{p-1} \lambda^{p} E\left[\sup _{0 \leq t \leq t_{1}}\left|\int_{0}^{t} h(x(s), x(s-\tau)) \mathrm{d} s\right|^{p}\right] \\
& \leq 2^{p-1} H \int_{0}^{t_{1}} E|h(x(s), x(s-\tau))|^{p} \mathrm{~d} s \\
& \quad+(2 T)^{p-1} \lambda^{p} \int_{0}^{t_{1}} E|h(x(s), x(s-\tau))|^{p} \mathrm{~d} s \\
& \leq(2 L)^{p / 2}\left[2^{p-1} H+(2 T)^{p-1} \lambda^{p}\right] \\
& \quad \times\left(T+\int_{0}^{t_{1}} E \sup _{-\tau \leq v \leq s}|x(\nu)|^{p} \mathrm{~d} s\right) .
\end{aligned}
$$

By the above inequalities, we can obtain

$$
\begin{aligned}
E\left(\sup _{-\tau \leq t \leq t_{1}}|x(t)|^{p}\right) & \\
\leq & E\|\xi\|^{p}+E\left[\sup _{0 \leq t \leq t_{1}}|x(t)|^{p}\right] \\
\leq & E\|\xi\|^{p}+6^{p-1} E\|\xi\|^{p}+6^{p-1} L_{p}\left(1+E\|\xi\|^{p}\right) \\
& +6^{p-1} L_{p}\left(1+E\left[\sup _{-\tau \leq t \leq t_{1}}|x(t)|^{p}\right]\right) \\
& +6^{p-1}\left(T^{p-1}\left(2 L_{d}\right)^{p / 2}+\left(2 L_{d}\right)^{p / 2} H\right. \\
& \left.+\left(2 L_{d}\right)^{p / 2} 2^{p-1} H+\left(2 L_{d}\right)^{p / 2}(2 T)^{p-1} \lambda^{p}\right) \\
& \times\left(T+\int_{0}^{t_{1}} E \sup _{-\tau \leq \nu \leq s}|x(\nu)|^{p} \mathrm{~d} s\right) ;
\end{aligned}
$$

that is,

$$
\begin{aligned}
& E\left(\sup _{-\tau \leq t \leq t_{1}}|x(t)|^{p}\right) \\
& \leq\left(2 L_{p} 6^{p-1}+\left(1+6^{p-1}\left(1+L_{p}\right)\right) E\|\xi\|^{p}\right. \\
&\left.\quad+6^{p-1}\left(2 L_{d}\right)^{p / 2}\left(T^{p}+\left(2^{p-1}+1\right) H T+T^{p} 2^{p-1} \lambda^{p}\right)\right) \\
& \quad \times\left(1-6^{p-1} L_{p}\right)^{-1} \\
&+\left(6^{p-1}\left(2 L_{d}\right)^{p / 2}\left(T^{p-1}+\left(2^{p-1}+1\right) H+(2 T)^{p-1} \lambda^{p}\right)\right) \\
& \quad \times\left(1-6^{p-1} L_{p}\right)^{-1} \\
& \quad \times \int_{0}^{t_{1}} E \sup _{-\tau \leq \nu \leq s}|x(\nu)|^{p} \mathrm{~d} s \\
& \leq c_{1}+c_{2} \int_{0}^{t_{1}} E \sup _{-\tau \leq \nu \leq s}|x(\nu)|^{p} \mathrm{~d} s,
\end{aligned}
$$

where

$$
\begin{aligned}
c_{1}= & \left(2 L_{p} 6^{p-1}+\left(1+6^{p-1}\left(1+L_{p}\right)\right) E\|\xi\|^{p}\right. \\
& \left.+6^{p-1}\left(2 L_{d}\right)^{p / 2}\left(T^{p}+\left(2^{p-1}+1\right) H T+T^{p} 2^{p-1} \lambda^{p}\right)\right) \\
& \times\left(1-6^{p-1} L_{p}\right)^{-1}, \\
c_{2}= & \left(6^{p-1}\left(2 L_{d}\right)^{p / 2}\left(T^{p-1}+\left(2^{p-1}+1\right) H+(2 T)^{p-1} \lambda^{p}\right)\right) \\
& \times\left(1-6^{p-1} L_{p}\right)^{-1} .
\end{aligned}
$$

The Gronwall inequality shows that

$$
E\left(\sup _{-\tau \leq t \leq T}|x(t)|^{p}\right) \leq c_{1} e^{c_{2} T} \equiv A_{1} .
$$

Then we can prove in the same way that the Euler-Maruyama approximate solution to (1) has the property that

$$
E\left(\sup _{-\tau \leq t \leq T}|y(t)|^{p}\right) \leq A_{2}
$$

So, we can obtain

$$
E\left(\sup _{-\tau \leq t \leq T}|x(t)|^{p}\right) \vee E\left(\sup _{-\tau \leq t \leq T}|y(t)|^{p}\right) \leq A,
$$

by letting $A=A_{1} \vee A_{2}$.

Lemma 3. Under (H1)-(H2),

$$
E\left(\sup _{-\tau \leq t \leq T}\left|y\left(t \wedge \rho_{d}\right)\right|^{2}\right)<C_{1}, \quad \forall t \in[-\tau, T],
$$

where $C_{1}$ is a positive constant independent of $\Delta$. 
Proof. For any $t_{1} \in[0, T]$, we have

$$
\begin{aligned}
& E\left(\sup _{-\tau \leq t \leq t_{1}}\left|y\left(t \wedge \rho_{d}\right)\right|^{2}\right) \\
& \quad \leq E\|\xi\|^{2}+E\left(\sup _{0 \leq t \leq t_{1}}\left|y\left(t \wedge \rho_{d}\right)\right|^{2}\right) .
\end{aligned}
$$

By (4) and inequality

$$
\begin{aligned}
& (a+b+c+d+e+f)^{2} \\
& \quad \leq 6\left(|a|^{2}+|b|^{2}+|c|^{2}+|d|^{2}+|e|^{2}+|f|^{2}\right)
\end{aligned}
$$

and (11) then we have

$$
\begin{aligned}
& E\left(\sup _{0 \leq t \leq t_{1}}\left|y\left(t \wedge \rho_{d}\right)\right|^{2}\right) \\
& \leq 6 E\left[\sup _{0 \leq t \leq t_{1}}\left|G\left(\tilde{y}\left(t \wedge \rho_{d}-\tau\right)\right)\right|^{2}\right] \\
& +6 E|\xi(0)|^{2}+6 E|G(\xi(-\tau))|^{2} \\
& +6 E\left[\sup _{0 \leq t \leq t_{1}}\left|\int_{0}^{t \wedge \rho_{d}} f(\tilde{y}(s), \tilde{y}(s-\tau)) \mathrm{d} s\right|^{2}\right] \\
& +6 E\left[\sup _{0 \leq t \leq t_{1}}\left|\int_{0}^{t \wedge \rho_{d}} g(\tilde{y}(s), \tilde{y}(s-\tau)) \mathrm{d} W(s)\right|^{2}\right] \\
& +6 E\left[\sup _{0 \leq t \leq t_{1}}\left|\int_{0}^{t \wedge \rho_{d}} h(\tilde{y}(s), \tilde{y}(s-\tau)) \mathrm{d} N(s)\right|^{2}\right] \\
& \leq 6 K_{1} E\left[1+\sup _{0 \leq t \leq t_{1}}\left|\tilde{y}\left(t \wedge \rho_{d}-\tau\right)\right|^{2}\right] \\
& +6 E\|\xi\|^{2}+6 K_{1}\left(1+E\|\xi\|^{2}\right) \\
& +6 E\left[\sup _{0 \leq t \leq t_{1}}\left|\int_{0}^{t \wedge \rho_{d}} f(\tilde{y}(s), \tilde{y}(s-\tau)) \mathrm{d} s\right|^{2}\right] \\
& +6 E\left[\sup _{0 \leq t \leq t_{1}}\left|\int_{0}^{t \wedge \rho_{d}} g(\tilde{y}(s), \tilde{y}(s-\tau)) \mathrm{d} W(s)\right|^{2}\right] \\
& +6 E\left[\sup _{0 \leq t \leq t_{1}}\left|\int_{0}^{t \wedge \rho_{d}} h(\tilde{y}(s), \tilde{y}(s-\tau)) \mathrm{d} N(s)\right|^{2}\right] \text {. }
\end{aligned}
$$

Using the Cauchy-Schwarz inequality, Fubini's Theorem, and (8),

$$
\begin{aligned}
& E\left[\sup _{0 \leq t \leq t_{1}}\left|\int_{0}^{t \wedge \rho_{d}} f(\tilde{y}(s), \tilde{y}(s-\tau)) \mathrm{d} s\right|^{2}\right] \\
& \quad \leq T E\left[\sup _{0 \leq t \leq t_{1}} \int_{0}^{t \wedge \rho_{d}}|f(\tilde{y}(s), \tilde{y}(s-\tau))|^{2} \mathrm{~d} s\right] \\
& \quad \leq T L_{d} E\left[\int_{0}^{t_{1} \wedge \rho_{d}}\left(1+|\tilde{y}(s)|^{2}+|\tilde{y}(s-\tau)|^{2}\right) \mathrm{d} s\right] \\
& \quad \leq T L_{d}\left(T+2 \int_{0}^{t_{1}} E\left(\sup _{-\tau \leq \nu \leq s \wedge \rho_{d}}|y(\nu)|^{2}\right) \mathrm{d} s\right) .
\end{aligned}
$$

Then by the Doob martingale inequality and (8), we have

$$
\begin{aligned}
& E\left[\sup _{0 \leq t \leq t_{1}}\left|\int_{0}^{t \wedge \rho_{d}} g(\tilde{y}(s), \tilde{y}(s-\tau)) \mathrm{d} W(s)\right|^{2}\right] \\
& \quad \leq 4 E\left[\int_{0}^{t_{1} \wedge \rho_{d}}|g(\tilde{y}(s), \tilde{y}(s-\tau))|^{2} \mathrm{~d} s\right] \\
& \quad \leq 4 L_{d} E\left[\int_{0}^{t_{1} \wedge \rho_{d}}\left(1+|\tilde{y}(s)|^{2}+|\tilde{y}(s-\tau)|^{2}\right) \mathrm{d} s\right] \\
& \quad \leq 4 L_{d}\left(T+2 \int_{0}^{t_{1}} E\left(\sup _{-\tau \leq \nu \leq s \wedge \rho_{d}}|y(\nu)|^{2}\right) \mathrm{d} s\right) .
\end{aligned}
$$

For the jump integral, we can transform to the compensated Poisson process

$$
\widetilde{N}(t)=N(t)-\lambda t
$$

which is a martingale, and use the isometry

$$
\begin{aligned}
& E\left[\left|\int_{a}^{b} h(\tilde{y}(s), \tilde{y}(s-\tau)) \mathrm{d} \widetilde{N}(s)\right|^{2}\right] \\
& =\lambda\left[\int_{a}^{b} E|h(\tilde{y}(s), \tilde{y}(s-\tau))|^{2} \mathrm{~d} s\right]
\end{aligned}
$$

to obtain

$$
\begin{aligned}
& E\left[\sup _{0 \leq t \leq t_{1}}\left|\int_{0}^{t \wedge \rho_{d}} h(\tilde{y}(s), \tilde{y}(s-\tau)) \mathrm{d} N(s)\right|^{2}\right] \\
& =E\left[\sup _{0 \leq t \leq t_{1}} \mid \int_{0}^{t \wedge \rho_{d}} h(\widetilde{y}(s), \widetilde{y}(s-\tau)) \mathrm{d} \widetilde{N}(s)\right. \\
& \left.+\left.\lambda \int_{0}^{t \wedge \rho_{d}} h(\tilde{y}(s), \tilde{y}(s-\tau)) \mathrm{d} s\right|^{2}\right] \\
& \leq 2 E\left[\sup _{0 \leq t \leq t_{1}}\left|\int_{0}^{t \wedge \rho_{d}} h(\tilde{y}(s), \tilde{y}(s-\tau)) \mathrm{d} \widetilde{N}(s)\right|^{2}\right] \\
& +2 \lambda^{2} E\left[\sup _{0 \leq t \leq t_{1}}\left|\int_{0}^{t \wedge \rho_{d}} h(\tilde{y}(s), \tilde{y}(s-\tau)) \mathrm{d} s\right|^{2}\right] \\
& \leq 8 E\left[\left|\int_{0}^{t_{1} \wedge \rho_{d}} h(\tilde{y}(s), \tilde{y}(s-\tau)) \mathrm{d} \widetilde{N}(s)\right|^{2}\right] \\
& +2 \lambda^{2} T E\left[\int_{0}^{t_{1} \wedge \rho_{d}}|h(\tilde{y}(s), \tilde{y}(s-\tau))|^{2} \mathrm{~d} s\right] \\
& \leq\left(8 \lambda+2 \lambda^{2} T\right) E\left[\int_{0}^{t_{1} \wedge \rho_{d}}|h(\tilde{y}(s), \tilde{y}(s-\tau))|^{2} \mathrm{~d} s\right] \\
& \leq\left(8 \lambda+2 \lambda^{2} T\right) L_{d}\left(T+2 \int_{0}^{t_{1}} E\left(\sup _{-\tau \leq \nu \leq s \wedge \rho_{d}}|y(\nu)|^{2}\right) \mathrm{d} s\right) \text {. }
\end{aligned}
$$


Inserting (33)-(37) into (32) gives

$$
\begin{aligned}
E\left(\sup _{0 \leq t \leq t_{1}}\left|y\left(t \wedge \rho_{d}\right)\right|^{2}\right) \\
\leq 6 K_{1} E\left[1+\sup _{0 \leq t \leq t_{1}}\left|\tilde{y}\left(t \wedge \rho_{d}-\tau\right)\right|^{2}\right] \\
\quad+6 E\|\xi\|^{2}+6 K_{1}\left(1+E\|\xi\|^{2}\right) \\
\quad+6\left(T+4+8 \lambda+2 \lambda^{2} T\right) L_{d} \\
\quad \times\left(T+2 \int_{0}^{t_{1}} E\left(\sup _{-\tau \leq \nu \leq s \wedge \rho_{d}}|y(\nu)|^{2}\right) \mathrm{d} s\right) .
\end{aligned}
$$

Substitute the above inequality into (30), the result is

$$
\begin{aligned}
& E\left(\sup _{-\tau \leq t \leq t_{1}}\left|y\left(t \wedge \rho_{d}\right)\right|^{2}\right) \\
& \leq E\|\xi\|^{2}+E\left(\sup _{0 \leq t \leq t_{1}}\left|y\left(t \wedge \rho_{d}\right)\right|^{2}\right) \\
& \leq 12 K_{1}+\left(6 K_{1}+7\right) E\|\xi\|^{2} \\
& +6 K_{1} E \sup _{0 \leq t \leq t_{1}}\left|\tilde{y}\left(t \wedge \rho_{d}-\tau\right)\right|^{2} \\
& +6\left(T+4+8 \lambda+2 \lambda^{2} T\right) L_{d} \\
& \times\left(T+2 \int_{0}^{t_{1}} E\left(\sup _{-\tau \leq \nu \leq s \wedge \rho_{d}}|y(\nu)|^{2}\right) \mathrm{d} s\right) \\
& \leq 12 K_{1}+\left(6 K_{1}+7\right) E\|\xi\|^{2} \\
& +6 K_{1} E \sup _{-\tau \leq t \leq t_{1}}\left|y\left(t \wedge \rho_{d}\right)\right|^{2} \\
& +6\left(T+4+8 \lambda+2 \lambda^{2} T\right) L_{d} T \\
& +12\left(T+4+8 \lambda+2 \lambda^{2} T\right) L_{d} \\
& \times \int_{0}^{t_{1}} E\left(\sup _{-\tau \leq \nu \leq s}\left|y\left(\nu \wedge \rho_{d}\right)\right|^{2}\right) \mathrm{d} s .
\end{aligned}
$$

The Gronwall inequality shows that

$$
\begin{aligned}
& E\left(\sup _{-\tau \leq t \leq T}\left|y\left(t \wedge \rho_{d}\right)\right|^{2}\right) \\
& \leq\left(12 K_{1}+\left(6 K_{1}+7\right) E\|\xi\|^{2}\right. \\
&\left.+6\left(T+4+8 \lambda+2 \lambda^{2} T\right) L_{d} T\right) \\
& \times\left(1-6 K_{1}\right)^{-1} \\
& \times e^{12\left(T+4+8 \lambda+2 \lambda^{2} T\right) L_{d} T /\left(1-6 K_{1}\right)} \\
& \equiv C_{1} .
\end{aligned}
$$

This is the desired result.
Corollary 4. Under (H1)-(H2),

$$
E|y(t)|^{2} \leq C_{2}, \quad \forall t \in\left[-\tau, T \wedge \rho_{d}\right],
$$

where $C_{2}$ is a positive constant independent of $\Delta$.

Lemma 5. Under (H1)-(H2), for any $t \in[0, T]$, there exists a constant $C_{3}>0$ independent of $\Delta$, such that

$$
\int_{0}^{t \wedge \rho_{d}} E|y(s)-\tilde{y}(s)|^{2} \mathrm{~d} s \leq \mathrm{C}_{3} \Delta
$$

Proof. For any $t \in\left[0, T \wedge \rho_{d}\right]$, there exists an integer $k$ such that $t \in\left[t_{k}, t_{k+1}\right)$. By the definition of $y(t)$ and $\tilde{y}(t)$, we have

$$
\begin{aligned}
y(t)= & G(\tilde{y}(t-\tau))+\xi(0)-G(\xi(-\tau)) \\
& +\int_{0}^{t} f(\tilde{y}(s), \tilde{y}(s-\tau)) \mathrm{d} s \\
& +\int_{0}^{t} g(\tilde{y}(s), \tilde{y}(s-\tau)) \mathrm{d} W(t) \\
& +\int_{0}^{t} h(\tilde{y}(s), \tilde{y}(s-\tau)) \mathrm{d} N(t), \\
\tilde{y}(t)= & G\left(y_{k-m}\right)+\xi(0)-G(\xi(-\tau)) \\
& +\int_{0}^{k \Delta} f(\tilde{y}(s), \tilde{y}(s-\tau)) \mathrm{d} s \\
& +\int_{0}^{k \Delta} g(\tilde{y}(s), \tilde{y}(s-\tau)) \mathrm{d} W(t) \\
& +\int_{0}^{k \Delta} h(\tilde{y}(s), \tilde{y}(s-\tau)) \mathrm{d} N(t) .
\end{aligned}
$$

It is clear that $\tilde{y}(t)=y_{k}$ and $\tilde{y}(t-\tau)=y_{k-m}$, and thus

$$
\begin{aligned}
y(t)-\tilde{y}(t)= & \int_{k \Delta}^{t} f(\tilde{y}(s), \tilde{y}(s-\tau)) \mathrm{d} s \\
& +\int_{k \Delta}^{t} g(\tilde{y}(s), \tilde{y}(s-\tau)) \mathrm{d} W(t) \\
& +\int_{k \Delta}^{t} h(\tilde{y}(s), \tilde{y}(s-\tau)) \mathrm{d} N(t) \\
= & f(\tilde{y}(s), \tilde{y}(s-\tau))\left(t-t_{k}\right) \\
& +g(\tilde{y}(s), \tilde{y}(s-\tau))\left(W(t)-W\left(t_{k}\right)\right) \\
& +h(\tilde{y}(s), \tilde{y}(s-\tau))\left(N(t)-N\left(t_{k}\right)\right) .
\end{aligned}
$$

Then applying the inequality $(a+b+c)^{2} \leq 3\left(|a|^{2}+|b|^{2}+|c|^{2}\right)$ and (8), we have

$$
\begin{aligned}
\mid y(t) & -\left.\tilde{y}(t)\right|^{2} \\
\leq & 3|f(\tilde{y}(s), \tilde{y}(s-\tau))|^{2} \Delta^{2} \\
& +3|g(\tilde{y}(s), \tilde{y}(s-\tau))|^{2}\left|W(t)-W\left(t_{k}\right)\right|^{2} \\
& +3|h(\tilde{y}(s), \tilde{y}(s-\tau))|^{2}\left|N(t)-N\left(t_{k}\right)\right|^{2}
\end{aligned}
$$




$$
\begin{aligned}
\leq & 3 L_{d}\left(1+|\tilde{y}(t)|^{2}+|\tilde{y}(t-\tau)|^{2}\right) \\
& \times\left(\Delta^{2}+\left|W(t)-W\left(t_{k}\right)\right|^{2}+\left|N(t)-N\left(t_{k}\right)\right|^{2}\right) .
\end{aligned}
$$

Then by Corollary 4 and the Lyapunov inequality [22]

$$
\begin{aligned}
& E \int_{0}^{t \wedge \rho_{d}}|y(s)-\tilde{y}(s)|^{2} \mathrm{~d} s \\
& \leq 3 L_{d} E \int_{0}^{t \wedge \rho_{d}}\left(1+|\tilde{y}(t)|^{2}+|\tilde{y}(t-\tau)|^{2}\right) \\
& \times\left(\Delta^{2}+\left|W(t)-W\left(t_{k}\right)\right|^{2}+\left|N(t)-N\left(t_{k}\right)\right|^{2}\right) \mathrm{d} s \\
& \leq 3 L_{d} \int_{0}^{t \wedge \rho_{d}} E\left(1+|\tilde{y}(t)|^{2}+|\tilde{y}(t-\tau)|^{2}\right) E \\
& \times\left(\Delta^{2}+\left|W(t)-W\left(t_{k}\right)\right|^{2}+\left|N(t)-N\left(t_{k}\right)\right|^{2}\right) \mathrm{d} s \\
& \leq 3 L_{d} E \int_{0}^{t \wedge \rho_{d}}\left(1+|\tilde{y}(t)|^{2}+|\tilde{y}(t-\tau)|^{2}\right) \\
& \times\left(\Delta^{2}+m \Delta+\lambda \Delta\right) \mathrm{d} s \\
& \leq 3 L_{d} T\left(1+2 C_{2}\right)(\Delta+m+\lambda) \Delta \\
& \leq C_{3} \Delta .
\end{aligned}
$$

Let $C_{3}=3 L_{d} T\left(1+2 C_{2}\right)(1+m+\lambda)$; the lemma is therefore complete.

\section{Main Result}

Theorem 6. Under assumptions (H1)-(H2), the Euler-Maruyama approximate solution (4) converges to the exact solution of the NSDDEwPJs (1) in the sense that

$$
\lim _{\Delta \rightarrow 0} E\left[\sup _{0 \leq t \leq T}|x(t)-y(t)|^{2}\right]=0 .
$$

Proof. Let $e(t)=x(t)-y(t)$; it is easy to see that

$$
\begin{aligned}
E\left[\sup _{0 \leq t \leq T}|e(t)|^{2}\right] & \\
= & E\left[\sup _{0 \leq t \leq T}|e(t)|^{2} I_{\left\{\sigma_{d}>T, v_{d}>T\right\}}\right] \\
& +E\left[\sup _{0 \leq t \leq T}|e(t)|^{2} I_{\left\{\sigma_{d} \leq T \text { or } v_{d} \leq T\right\}}\right] \\
= & E\left[\sup _{0 \leq t \leq T}|e(t)|^{2} I_{\left\{\rho_{d}>T\right\}}\right] \\
& +E\left[\sup _{0 \leq t \leq T}|e(t)|^{2} I_{\left\{\sigma_{d} \leq T \text { or } v_{d} \leq T\right\}}\right] \\
\leq & E\left[\sup _{0 \leq t \leq T}\left|e\left(t \wedge \rho_{d}\right)\right|^{2}\right] \\
& +E\left[\sup _{0 \leq t \leq T}|e(t)|^{2} I_{\left\{\sigma_{d} \leq T \text { or } v_{d} \leq T\right\}}\right],
\end{aligned}
$$

where $I_{A}$ is the indicator function of set $A$.
Recall the Young inequality, for $1 / p+1 / q=1(p, q>0)$; we have

$$
\begin{aligned}
a b & \leq a \delta^{1 / p} \frac{b}{\delta^{1 / p}} \leq \frac{\left(a \delta^{1 / p}\right)^{p}}{p}+\frac{b^{q}}{q \delta^{q / p}} \\
& =\frac{a^{p} \delta}{p}+\frac{b^{q}}{q \delta^{q / p}}, \quad \forall a, b, \delta>0 .
\end{aligned}
$$

Thus for any $\delta>0$, we have

$$
\begin{aligned}
& E\left[\sup _{0 \leq t \leq T}|e(t)|^{2} I_{\left\{\sigma_{d} \leq T \text { or } v_{d} \leq T\right\}}\right] \\
& \quad \leq \frac{2 \delta}{p} E\left[\sup _{0 \leq t \leq T}|e(t)|^{p}\right]+\frac{1-2 / p}{\delta^{2 /(P-2)}} P\left\{\sigma_{d} \leq T \text { or } v_{d} \leq T\right\} .
\end{aligned}
$$

By Lemma 2, then

$$
\begin{aligned}
P\left\{\sigma_{d} \leq T\right\} & =E\left[I_{\left\{\sigma_{d} \leq T\right\}} \frac{\left|y\left(\sigma_{d}\right)\right|^{p}}{d^{p}}\right] \\
& \leq \frac{1}{d^{P}} E\left[\sup _{0 \leq t \leq T}|y(t)|^{p}\right] \leq \frac{A}{d^{p}} .
\end{aligned}
$$

Similarly, the result can be derived for $v_{d}$ as

$$
P\left\{v_{d} \leq T\right\} \leq \frac{A}{d^{p}}
$$

so that

$$
P\left\{\sigma_{d} \leq T \text { or } v_{d} \leq T\right\} \leq P\left\{\sigma_{d} \leq T\right\}+P\left\{v_{d} \leq T\right\} \leq \frac{2 A}{d^{p}} .
$$

Using these bounds along with

$$
\begin{aligned}
& E\left[\sup _{0 \leq t \leq T}|e(t)|^{p}\right] \\
& \quad \leq 2^{p-1}\left(E\left[\sup _{0 \leq t \leq T}|x(t)|^{p}\right]+E\left[\sup _{0 \leq t \leq T}|y(t)|^{p}\right]\right) \\
& \quad \leq 2^{p} A
\end{aligned}
$$

in (50) gives

$$
E\left[\sup _{0 \leq t \leq T}|e(t)|^{2} I_{\left\{\sigma_{d} \leq T \text { or } v_{d} \leq T\right\}}\right] \leq \frac{2^{p+1} \delta A}{p}+\frac{2(P-2) A}{P \delta^{2 /(P-2)} d^{p}} .
$$

Now we bound the first term on the right-hand side of (48). By the definition of $x(t)$ and $y(t)$, we have

$$
\begin{aligned}
x(t & \left.\wedge \rho_{d}\right)-y\left(t \wedge \rho_{d}\right) \\
= & G\left(x\left(t \wedge \rho_{d}-\tau\right)\right)-G\left(\tilde{y}\left(t \wedge \rho_{d}-\tau\right)\right) \\
& +\int_{0}^{t \wedge \rho_{d}} f(x(s), x(s-\tau))-f(\tilde{y}(s), \tilde{y}(s-\tau)) \mathrm{d} s
\end{aligned}
$$




$$
\begin{aligned}
& +\int_{0}^{t \wedge \rho_{d}} g(x(s), x(s-\tau))-g(\tilde{y}(s), \tilde{y}(s-\tau)) \mathrm{d} W(s) \\
& +\int_{0}^{t \wedge \rho_{d}} h(x(s), x(s-\tau))-h(\tilde{y}(s), \tilde{y}(s-\tau)) \mathrm{d} N(s) .
\end{aligned}
$$

For ease of exposition, we abbreviate

$$
\begin{aligned}
& \widetilde{G}(s)=G(x(s-\tau))-G(\tilde{y}(s-\tau)), \\
& \tilde{f}(s)=f(x(s), x(s-\tau))-f(\tilde{y}(s), \tilde{y}(s-\tau)), \\
& \tilde{g}(s)=g(x(s), x(s-\tau))-g(\tilde{y}(s), \tilde{y}(s-\tau)), \\
& \tilde{h}(s)=h(x(s), x(s-\tau))-h(\tilde{y}(s), \tilde{y}(s-\tau)) .
\end{aligned}
$$

Thus, for any $t \in[0, T]$,

$$
\begin{aligned}
& E \sup _{0 \leq t \leq t_{1}}\left|x\left(t \wedge \rho_{d}\right)-y\left(t \wedge \rho_{d}\right)\right|^{2} \\
& \leq 4 E \sup _{0 \leq t \leq t_{1}}|\widetilde{G}(t)|^{2}+4 E \sup _{0 \leq t \leq t_{1}}\left|\int_{0}^{t \wedge \rho_{d}} \widetilde{f}(s) \mathrm{d} s\right|^{2} \\
& \quad+4 E \sup _{0 \leq t \leq t_{1}}\left|\int_{0}^{t \wedge \rho_{d}} \widetilde{g}(s) \mathrm{d} W(s)\right|^{2} \\
& \quad+4 E \sup _{0 \leq t \leq t_{1}}\left|\int_{0}^{t \wedge \rho_{d}} \widetilde{h}(s) \mathrm{d} N(s)\right|^{2}
\end{aligned}
$$

By the process of Lemma 5 and (45), we can get

$$
\begin{aligned}
& E \sup _{0 \leq t \leq t_{1}}\left|y\left(t \wedge \rho_{d}\right)-\tilde{y}\left(t \wedge \rho_{d}\right)\right|^{2} \\
& \quad \leq 3 L_{d}\left(1+2 C_{2}\right)\left(\Delta^{2}+m \Delta+\lambda \Delta\right) .
\end{aligned}
$$

By condition (H2) and (59), we have

$$
\begin{aligned}
E \sup _{0 \leq t \leq t_{1}}\left|G\left(x\left(t \wedge \rho_{d}-\tau\right)\right)-G\left(\tilde{y}\left(t \wedge \rho_{d}-\tau\right)\right)\right|^{2} \\
\leq 2 E \sup _{0 \leq t \leq t_{1}}\left|G\left(x\left(t \wedge \rho_{d}-\tau\right)\right)-G\left(y\left(t \wedge \rho_{d}-\tau\right)\right)\right|^{2} \\
\quad+2 E \sup _{0 \leq t \leq t_{1}}\left|G\left(y\left(t \wedge \rho_{d}-\tau\right)\right)-G\left(\tilde{y}\left(t \wedge \rho_{d}-\tau\right)\right)\right|^{2} \\
\leq 2 K^{2} E \sup _{0 \leq t \leq t_{1}}\left|x\left(t \wedge \rho_{d}-\tau\right)-y\left(t \wedge \rho_{d}-\tau\right)\right|^{2} \\
\quad+2 K^{2} E \sup _{0 \leq t \leq t_{1}}\left|y\left(t \wedge \rho_{d}-\tau\right)-\tilde{y}\left(t \wedge \rho_{d}-\tau\right)\right|^{2} \\
\leq 2 K^{2} E \sup _{0 \leq t \leq t_{1}}\left|x\left(t \wedge \rho_{d}-\tau\right)-y\left(t \wedge \rho_{d}-\tau\right)\right|^{2} \\
\quad+6 K^{2} L_{d}\left(1+2 C_{2}\right)\left(\Delta^{2}+m \Delta+\lambda \Delta\right) \\
\leq 2 K^{2} E \sup _{0 \leq t \leq t_{1}}\left|x\left(t \wedge \rho_{d}\right)-y\left(t \wedge \rho_{d}\right)\right|^{2} \\
+6 K^{2} L_{d}\left(1+2 C_{2}\right)\left(\Delta^{2}+m \Delta+\lambda \Delta\right) .
\end{aligned}
$$

By Cauchy-Schwarz inequality and condition (H1) and Lemma 5, we get

$$
\begin{aligned}
E \sup _{0 \leq t \leq t_{1}} \mid \int_{0}^{t \wedge \rho_{d}} f(x(s), x(s-\tau)) & \quad-\left.f(\tilde{y}(s), \tilde{y}(s-\tau)) \mathrm{d} s\right|^{2} \\
\leq & T E \int_{0}^{t_{1} \wedge \rho_{d}}|f(x(s), x(s-\tau))-f(\tilde{y}(s), \tilde{y}(s-\tau))|^{2} \mathrm{~d} s \\
\leq & T C_{d} E \\
& \times \int_{0}^{t_{1} \wedge \rho_{d}}\left(|x(s)-\tilde{y}(s)|^{2}+|x(s-\tau)-\tilde{y}(s-\tau)|^{2}\right) \mathrm{d} s \\
\leq & 4 T C_{d} E \int_{0}^{t_{1} \wedge \rho_{d}}\left(|x(s)-y(s)|^{2}+|y(s)-\tilde{y}(s)|^{2}\right) \mathrm{d} s \\
\leq & 4 T C_{d} \int_{0}^{t_{1}} E\left[\sup _{0 \leq \nu \leq s}\left|x\left(\nu \wedge \rho_{d}\right)-y\left(\nu \wedge \rho_{d}\right)\right|^{2}\right] \mathrm{d} s \\
& +4 T C_{d} C_{3} \Delta .
\end{aligned}
$$

Similarly, by Burkholder-Davis-Gundy inequality and (8), we have

$$
\begin{aligned}
E \sup _{0 \leq t \leq t_{1}} \mid \int_{0}^{t \wedge \rho_{d}} g(x(s), x(s-\tau)) & -\left.g(\tilde{y}(s), \tilde{y}(s-\tau)) \mathrm{d} W(s)\right|^{2} \\
\leq & 4 E \int_{0}^{t_{1} \wedge \rho_{d}}|g(x(s), x(s-\tau))-g(\tilde{y}(s), \tilde{y}(s-\tau))|^{2} \mathrm{~d} s \\
\leq & 4 C_{d} E \\
& \times \int_{0}^{t_{1} \wedge \rho_{d}}\left(|x(s)-\tilde{y}(s)|^{2}+|x(s-\tau)-\tilde{y}(s-\tau)|^{2}\right) \mathrm{d} s \\
\leq & 16 C_{d} E \int_{0}^{t_{1} \wedge \rho_{d}}\left(|x(s)-y(s)|^{2}+|y(s)-\tilde{y}(s)|^{2}\right) \mathrm{d} s \\
\leq & 16 C_{d} \int_{0}^{t_{1}} E\left[\sup _{0 \leq \nu \leq s}\left|x\left(\nu \wedge \rho_{d}\right)-y\left(\nu \wedge \rho_{d}\right)\right|^{2}\right] \mathrm{d} s \\
& +16 C_{d} C_{3} \Delta .
\end{aligned}
$$

Then by (37) and condition (H1) and Lemma 5, we get

$$
\begin{aligned}
& E \sup _{0 \leq t \leq t_{1}}\left|\int_{0}^{t \wedge \rho_{d}} \tilde{h}(x(s), x(s-\tau)) \mathrm{d} N(s)\right|^{2} \\
& \quad \leq\left(8 \lambda+2 \lambda^{2} T\right) E\left[\int_{0}^{t_{1} \wedge \rho_{d}}|\widetilde{h}(\tilde{y}(s), \tilde{y}(s-\tau))|^{2} \mathrm{~d} s\right]
\end{aligned}
$$




$$
\begin{aligned}
= & \left(8 \lambda+2 \lambda^{2} T\right) E \\
& \times\left[\int_{0}^{t_{1} \wedge \rho_{d}}|h(x(s), x(s-\tau))-h(\tilde{y}(s), \tilde{y}(s-\tau))|^{2} \mathrm{~d} s\right] \\
\leq & \left(8 \lambda+2 \lambda^{2} T\right) C_{d} E \\
& \times \int_{0}^{t_{1} \wedge \rho_{d}}\left(|x(s)-\tilde{y}(s)|^{2}+|x(s-\tau)-\tilde{y}(s-\tau)|^{2}\right) \mathrm{d} s \\
\leq & 4\left(8 \lambda+2 \lambda^{2} T\right) C_{d} E \\
& \times \int_{0}^{t_{1} \wedge \rho_{d}}\left(|x(s)-y(s)|^{2}+|y(s)-\tilde{y}(s)|^{2}\right) \mathrm{d} s \\
\leq & 4\left(8 \lambda+2 \lambda^{2} T\right) C_{d} \\
& \times \int_{0}^{t_{1}} E\left[\sup _{0 \leq v \leq s}\left|x\left(\nu \wedge \rho_{d}\right)-y\left(\nu \wedge \rho_{d}\right)\right|^{2}\right] \mathrm{d} s \\
& +4\left(8 \lambda+2 \lambda^{2} T\right) C_{d} C_{3} \Delta .
\end{aligned}
$$

Taking (60)- (63) into (58), we obtain

$$
\begin{aligned}
& E \sup _{0 \leq t \leq t_{1}}\left|x\left(t \wedge \rho_{d}\right)-y\left(t \wedge \rho_{d}\right)\right|^{2} \\
& \leq 8 K^{2} E \sup _{0 \leq t \leq t_{1}}\left|x\left(t \wedge \rho_{d}\right)-y\left(t \wedge \rho_{d}\right)\right|^{2} \\
&+24 K^{2} L_{d}\left(1+2 C_{2}\right)\left(\Delta^{2}+m \Delta+\lambda \Delta\right) \\
&+16 T C_{d} \int_{0}^{t_{1}} E\left[\sup _{0 \leq v \leq s}\left|x\left(\nu \wedge \rho_{d}\right)-y\left(\nu \wedge \rho_{d}\right)\right|^{2}\right] \mathrm{d} s \\
&+16 T C_{d} C_{3} \Delta \\
&+64 C_{d} \int_{0}^{t_{1}} E\left[\sup _{0 \leq \nu \leq s}\left|x\left(\nu \wedge \rho_{d}\right)-y\left(\nu \wedge \rho_{d}\right)\right|^{2}\right] \mathrm{d} s \\
&+64 C_{d} C_{3} \Delta+16\left(8 \lambda+2 \lambda^{2} T\right) C_{d} \\
& \times \int_{0}^{t_{1}} E\left[\sup _{0 \leq v \leq s}\left|x\left(\nu \wedge \rho_{d}\right)-y\left(\nu \wedge \rho_{d}\right)\right|^{2}\right] \mathrm{d} s \\
&+16\left(8 \lambda+2 \lambda^{2} T\right) C_{d} C_{3} \Delta .
\end{aligned}
$$

Therefore

$$
\begin{aligned}
& E \sup _{0 \leq t \leq t_{1}}\left|e\left(t \wedge \rho_{d}\right)\right|^{2} \\
& \leq(\left(24 K^{2} L_{d}\left(1+2 C_{2}\right)(\Delta+m+\lambda)\right. \\
&\left.+\left[16 T+64+16\left(8 \lambda+2 \lambda^{2} T\right)\right] C_{d} C_{3}\right) \\
&\left.\times\left(1-8 K^{2}\right)^{-1}\right) \Delta
\end{aligned}
$$

$$
\begin{aligned}
& +\frac{\left[16 T+64+16\left(8 \lambda+2 \lambda^{2} T\right)\right] C_{d}}{1-8 K^{2}} \\
& \times \int_{0}^{t_{1}} E\left[\sup _{0 \leq \nu \leq s}\left|x\left(\nu \wedge \rho_{d}\right)-y\left(\nu \wedge \rho_{d}\right)\right|^{2}\right] \mathrm{d} s .
\end{aligned}
$$

That is, for $K^{2}<1 / 8$

$$
E \sup _{0 \leq t \leq T}\left|e\left(t \wedge \rho_{d}\right)\right|^{2} \leq C_{4} e^{\left[16 T+64+16\left(8 \lambda+2 \lambda^{2} T\right)\right] T C_{d} /\left(1-8 K^{2}\right)} \Delta,
$$

where

$$
\begin{aligned}
C_{4}= & \left(24 K^{2} L_{d}\left(1+2 C_{2}\right)(\Delta+m+\lambda)\right. \\
& \left.+\left[16 T+64+16\left(8 \lambda+2 \lambda^{2} T\right)\right] C_{d} C_{3}\right) \\
& \times\left(1-8 K^{2}\right)^{-1} .
\end{aligned}
$$

Taking (55) and (66) into (48), we have

$$
\begin{aligned}
E\left[\sup _{0 \leq t \leq T}|e(t)|^{2}\right] \leq & E\left[\sup _{0 \leq t \leq T}\left|e\left(t \wedge \rho_{d}\right)\right|^{2}\right] \\
& +E\left[\sup _{0 \leq t \leq T}|e(t)|^{2} I_{\left\{\sigma_{d}<T \text { or } v_{d}<T\right\}}\right] \\
\leq & C_{4} e^{\left[16 T+64+16\left(8 \lambda+2 \lambda^{2} T\right)\right] T C_{d} /\left(1-8 K^{2}\right)} \Delta \\
& +\frac{2^{p+1} \delta A}{p}+\frac{2(P-2) A}{P \delta^{2 /(P-2)} d^{p}} .
\end{aligned}
$$

Given any $\varepsilon>0$, we can choose $\delta$ sufficiently small for

$$
\frac{2^{p+1} \delta A}{p}<\frac{\varepsilon}{3}
$$

and then choose $d$ sufficient large for

$$
\frac{2(P-2) A}{P \delta^{2 /(P-2)} d^{p}}<\frac{\varepsilon}{3},
$$

and finally choose $\Delta$ so that

$$
C_{4} e^{\left[16 T+64+16\left(8 \lambda+2 \lambda^{2} T\right)\right] T C_{d} /\left(1-8 K^{2}\right)} \Delta<\frac{\varepsilon}{3} .
$$

Thus, $E\left[\sup _{0 \leq t \leq T}|e(t)|^{2}\right]<\varepsilon$. The proof is completed.

\section{Numerical Examples}

In this section, we present some numerical examples in support of our previous theoretical results.

Example 1. First we consider the following linear NSDDEwPJs:

$$
\begin{gathered}
\mathrm{d}[x(t)-0.5 x(t-1)] \\
=[-9 x(t)+7 x(t-1)] \mathrm{d} t+x(t-1) \mathrm{d} W(t) \\
+0.5 x(t-1) \mathrm{d} N(t), \quad t \in[0, T], \\
x(t)=t+1, \quad t \in[-1,0] .
\end{gathered}
$$




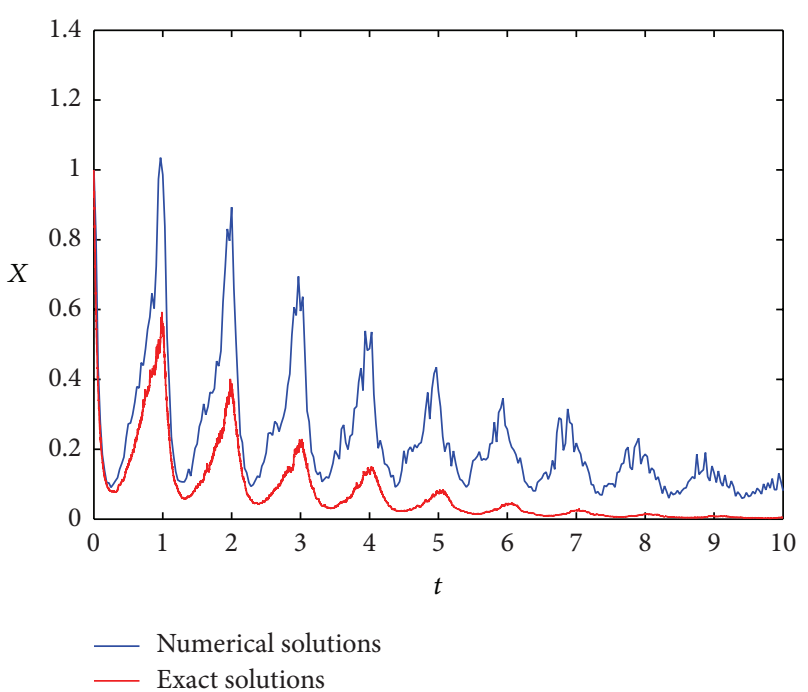

FIGURE 1: The exact solution and the numerical method approximations for Example 1.

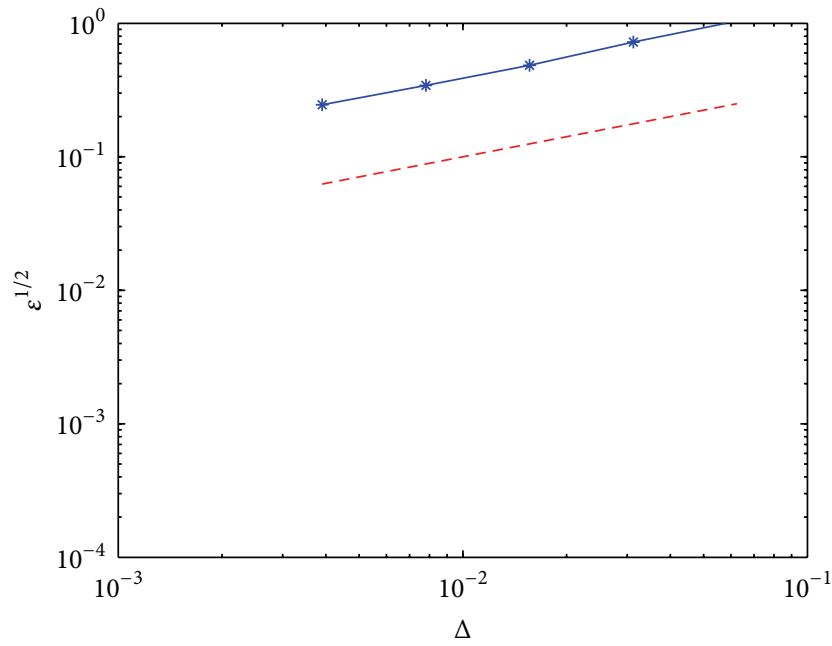

Figure 2: The convergence rate of the Euler-Maruyama method for Example 1.

First, we illustrate the strong convergence of the EulerMaruyama method for NSDDEs with Poisson jumps. We choose $T=10$ and $\lambda=10$. To the best of our knowledge, there are not any analytical solutions available for Example 1. Therefore, we use the Euler-Maruyama method to compute an "explicit solution" with step-size $\Delta=2^{-10}$ in our experiments. We draw the numerical solution obtained from the Euler-Maruyama method with step-size $\Delta=2^{-6}$ in Figure 1. The data used in the figure is obtained by the mean square of data by 1000 trajectories; that is $\omega_{i}: 1 \leq i \leq 1000$; $y_{n}=1 / 1000 \sum_{i=1}^{1000}\left|y_{n}\left(\omega_{i}\right)\right|^{2} ; t_{n}$ denotes the mesh point. From the figure, we can see that the exact solutions and numerical solutions match well.

To show the strong convergence order of the EulerMaruyama method, we apply the Euler-Maruyama method to Example 1. Then simulating the numerical solutions with
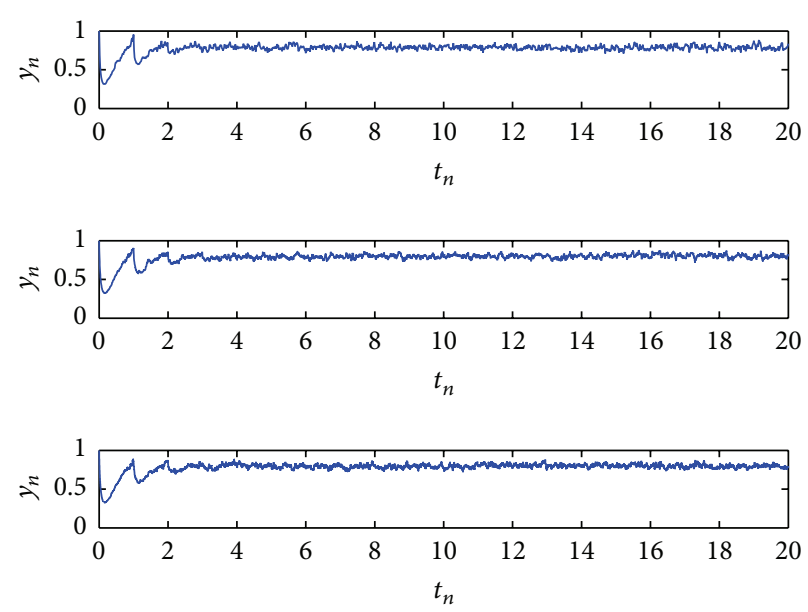

FIgURE 3: The Euler-Maruyama method approximations for Example 2 with step-sizes $\Delta=2^{-6}$ (upper), $\Delta=2^{-8}$ (middle), and $\Delta=2^{-10}$ (lower).

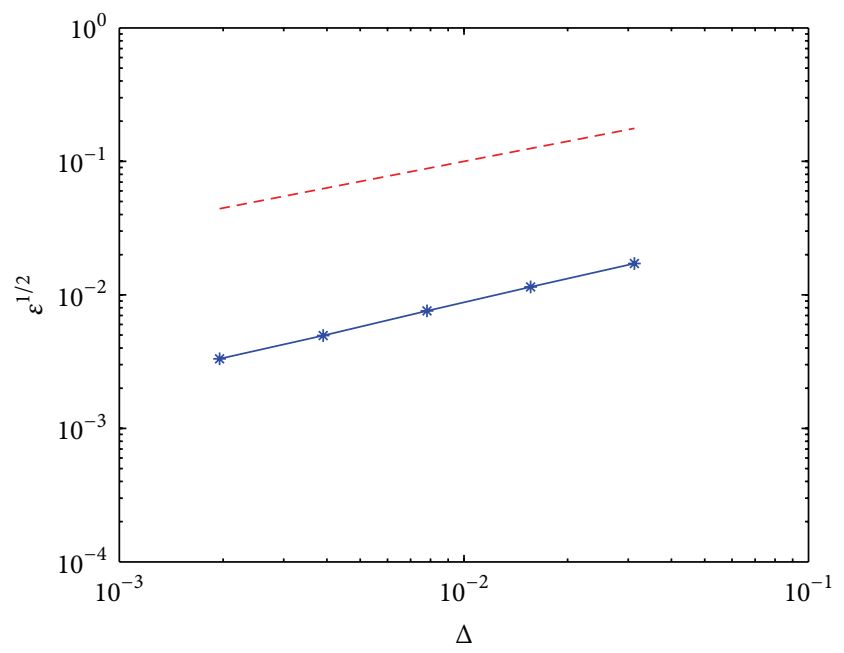

FIgURE 4: The convergence rate of the Euler-Maruyama method for Example 2.

5 different step-sizes $h=2^{p-1} \Delta$ for $1 \leq p \leq 5, \Delta=2^{-14}$. The mean-square errors $\varepsilon=1 / 1000 \sum_{i=1}^{1000}\left|y_{n}\left(\omega_{i}\right)-x(T)\right|^{2}$ all measured at time $T=10$ are estimated by trajectory averaging. We plot our approximation to $\sqrt{\varepsilon}$ against $\Delta$ on a $\log$-log scale in Figure 2. For reference a dashed line of slope one-half is added. It clearly shows that the Euler-Maruyama method for Example 1 is convergent with order 1/2.

Example 2. Consider the following nonlinear NSDDEwPJs:

$$
\begin{aligned}
& \mathrm{d}[x(t)-0.5 x(t-\tau)] \\
& =\left[-12 x^{3}(t)+10 x(t-1)\right] \mathrm{d} t+x(t-1) \mathrm{d} W(t) \\
& +0.1 x(t-1) \mathrm{d} N(t), \quad t \in[0, T] \\
& x(t)=t+1, \quad t \in[-\tau, 0]
\end{aligned}
$$


We fix $T=20$ and $\lambda=1$ for Example 2. The same as Figure 1, the data used in Figure 3 is obtained by the mean square of data by 1000 trajectories. In Figure 3, we show the numerical simulation of Example 2 by Euler-Maruyama method at step-sizes $\Delta=2^{-6}$ (upper), $\Delta=2^{-8}$ (middle), $\Delta=2^{-10}$ (lower).

Figure 4 also illustrates that the Euler-Maruyama method for Example 2 is convergent with order $1 / 2$.

\section{Conflict of Interests}

The authors declare that there is no conflict of interests regarding the publication of this paper.

\section{Acknowledgments}

This research was supported with funds provided by the National Natural Science Foundation of China (nos. 11226321, 11272229 , and 11102132). The authors thank the two anonymous reviewers for their very valuable comments and helpful suggestions which improve this paper significantly.

\section{References}

[1] V. Kolmanovskii, N. Koroleva, T. Maizenberg, X. Mao, and A. Matasov, "Neutral stochastic differential delay equations with Markovian switching," Stochastic Analysis and Applications, vol. 21, no. 4, pp. 819-847, 2003.

[2] V. B. Kolmanovskii and V. R. Nosov, Stability and Periodic Modes of Control Systems with Aftereffect, Nauka, Moscow, Russia, 1981.

[3] X. R. Mao, "Exponential stability in mean square of neutral stochastic differential-functional equations," Systems \& Control Letters, vol. 26, no. 4, pp. 245-251, 1995.

[4] X. R. Mao, "Razumikhin-type theorems on exponential stability of neutral stochastic functional-differential equations," SIAM Journal on Mathematical Analysis, vol. 28, no. 2, pp. 389-401, 1997.

[5] X. R. Mao, Stochastic Differential Equations and Their Applications, Horwood, Chichester, UK, 1997.

[6] X. R. Mao, Y. Shen, and C. G. Yuan, "Almost surely asymptotic stability of neutral stochastic differential delay equations with Markovian switching," Stochastic Processes and Their Applications, vol. 118, no. 8, pp. 1385-1406, 2008.

[7] Q. Luo, X. R. Mao, and Y. Shen, "New criteria on exponential stability of neutral stochastic differential delay equations," Systems \& Control Letters, vol. 55, no. 10, pp. 826-834, 2006.

[8] S. B. Zhou and S. G. Hu, "Razumikhin-type theorems of neutral stochastic functional differential equations," Acta Mathematica Scientia B, vol. 29, no. 1, pp. 181-190, 2009.

[9] J. W. Luo, "Fixed points and stability of neutral stochastic delay differential equations," Journal of Mathematical Analysis and Applications, vol. 334, no. 1, pp. 431-440, 2007.

[10] F. K. Wu and X. R. Mao, "Numerical solutions of neutral stochastic functional differential equations," SIAM Journal on Numerical Analysis, vol. 46, no. 4, pp. 1821-1841, 2008.

[11] H. M. Zhang and S. Q. Gan, "Mean square convergence of onestep methods for neutral stochastic differential delay equations," Applied Mathematics and Computation, vol. 204, no. 2, pp. 884890, 2008.
[12] S. B. Zhou and F. K. Wu, "Convergence of numerical solutions to neutral stochastic delay differential equations with Markovian switching," Journal of Computational and Applied Mathematics, vol. 229, no. 1, pp. 85-96, 2009.

[13] L. S. Wang, C. L. Mei, and H. Xue, "The semi-implicit Euler method for stochastic differential delay equations with jumps," Applied Mathematics and Computation, vol. 192, no. 2, pp. 567$578,2007$.

[14] L. S. Wang and H. Xue, "Convergence of numerical solutions to stochastic differential delay equations with Poisson jump and Markovian switching," Applied Mathematics and Computation, vol. 188, no. 2, pp. 1161-1172, 2007.

[15] R. H. Li and Z. G. Chang, "Convergence of numerical solution to stochastic delay differential equation with Poisson jump and Markovian switching," Applied Mathematics and Computation, vol. 184, no. 2, pp. 451-463, 2007.

[16] R. H. Li, H. B. Meng, and Y. H. Dai, "Convergence of numerical solutions to stochastic delay differential equations with jumps," Applied Mathematics and Computation, vol. 172, no. 1, pp. 584$602,2006$.

[17] J. W. Luo, "Comparison principle and stability of Ito stochastic differential delay equations with Poisson jump and Markovian switching," Nonlinear Analysis: Theory, Methods \& Applications, vol. 64, no. 2, pp. 253-262, 2006.

[18] L. Q. Cen and S. B. Zhou, "Convergence of numerical solutions to neutral stochastic delay differential equation with Poisson jump and Markovian switching," Journal of Applied Mathematics, vol. 23, no. 1, pp. 219-227, 2010.

[19] D. Z. Liu, G. Y. Yang, and W. Zhang, "The stability of neutral stochastic delay differential equations with Poisson jumps by fixed points," Journal of Computational and Applied Mathematics, vol. 235, no. 10, pp. 3115-3120, 2011.

[20] J. W. Luo and T. Taniguchi, "The existence and uniqueness for non-Lipschitz stochastic neutral delay evolution equations driven by Poisson jumps," Stochastics and Dynamics, vol. 9, no. 1, pp. 135-152, 2009.

[21] J. G. Tan, H. L. Wang, and Y. F. Guo, "Existence and uniqueness of solutions to neutral stochastic functional differential equations with Poisson jumps," Abstract and Applied Analysis, vol. 2012, Article ID 371239, 20 pages, 2012.

[22] P. E. Kloeden and E. Platen, Numerical Solution of Stochastic Differential Equations, Springer, Berlin, Germany, 1999. 


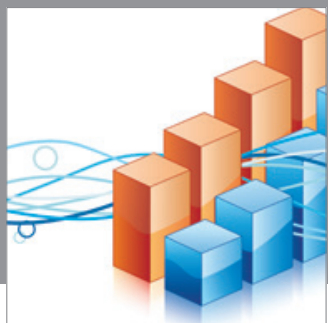

Advances in

Operations Research

mansans

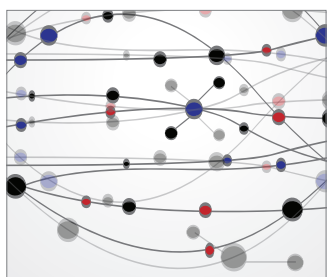

The Scientific World Journal
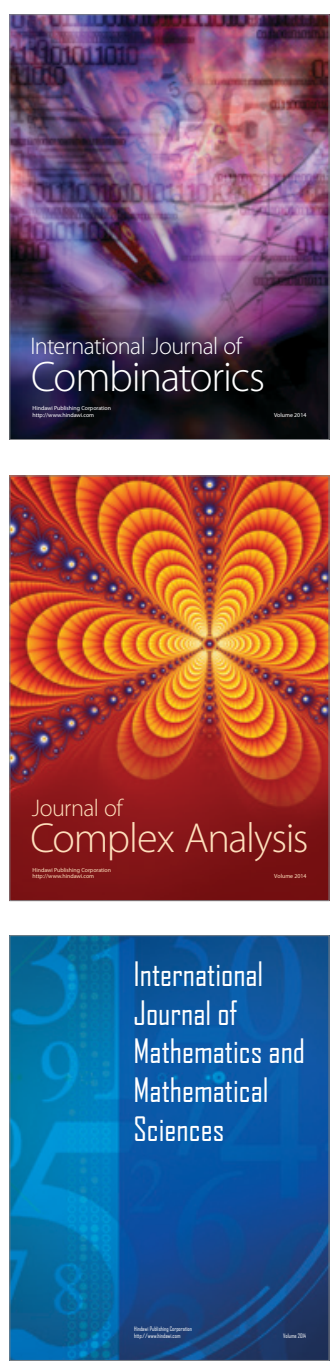
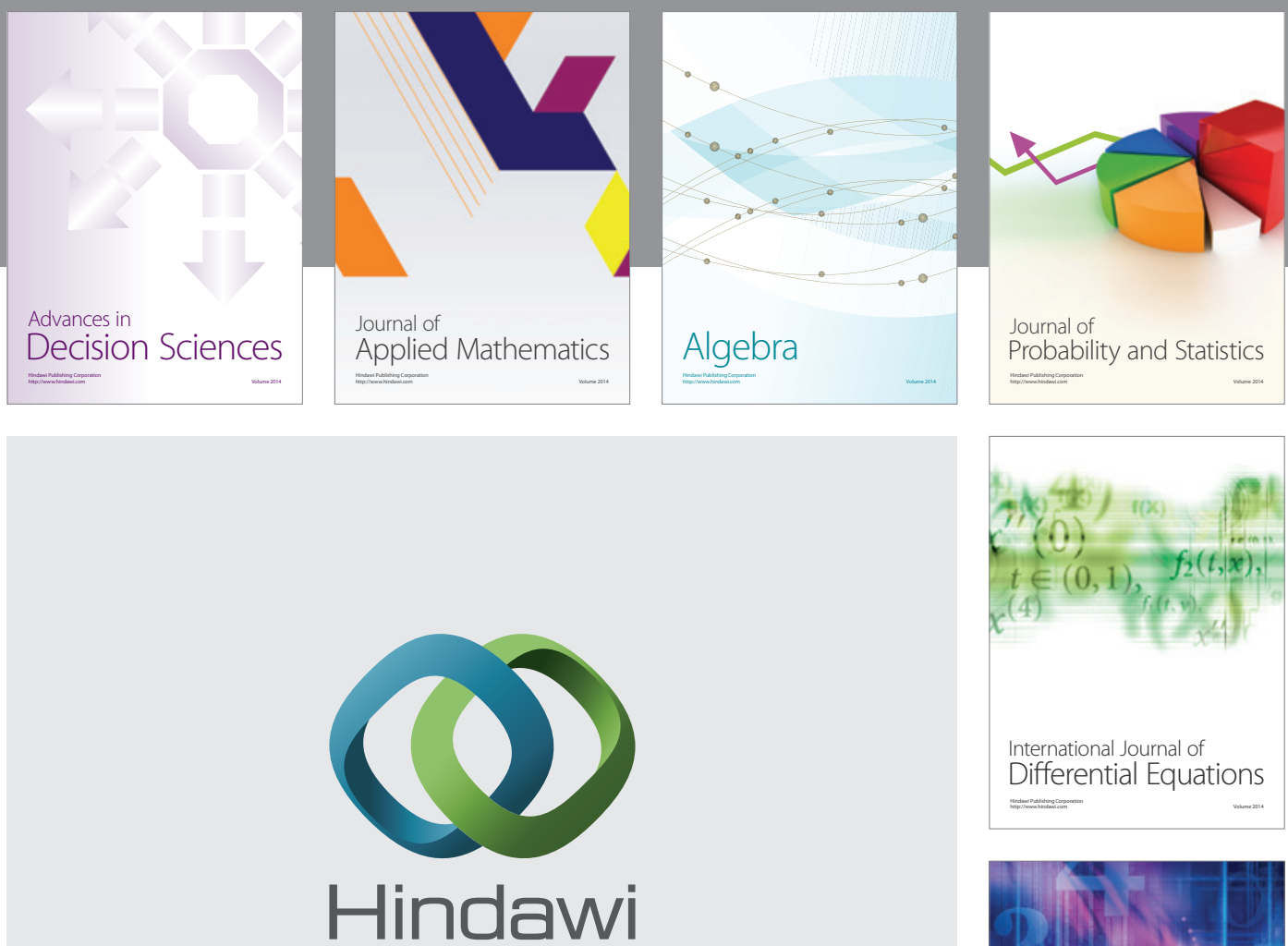

Submit your manuscripts at http://www.hindawi.com
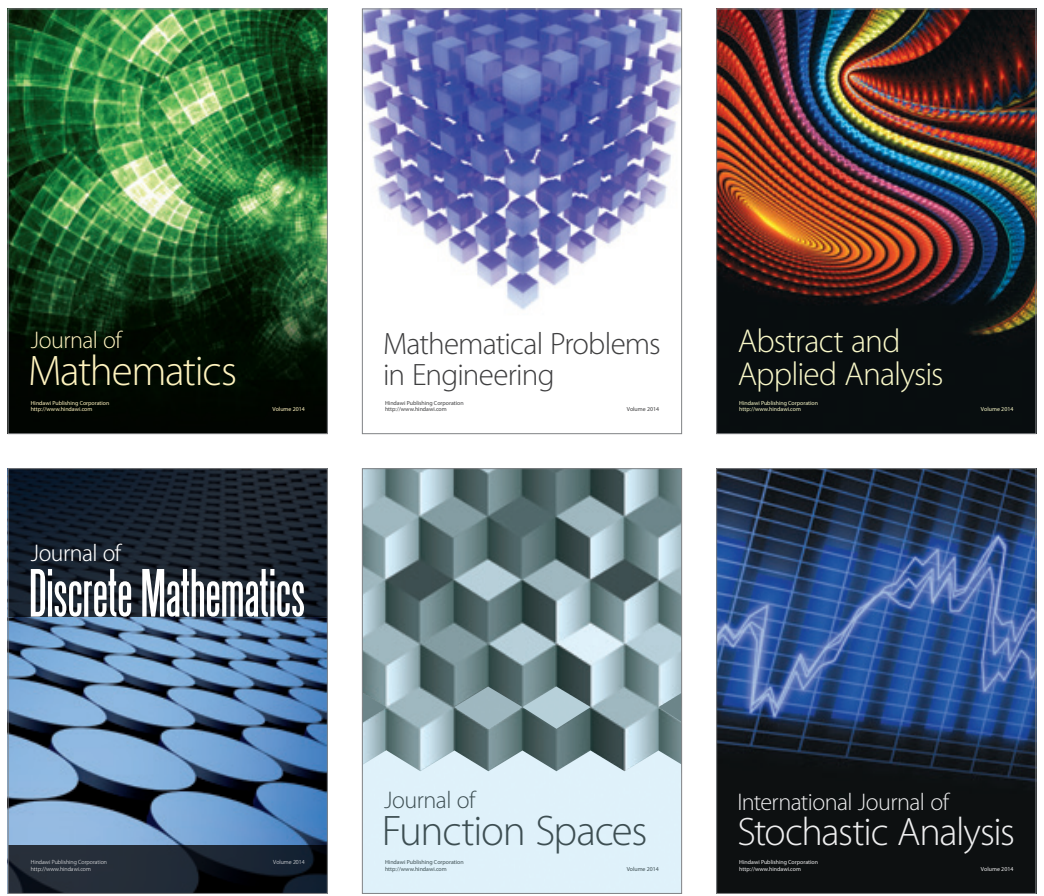

Journal of

Function Spaces

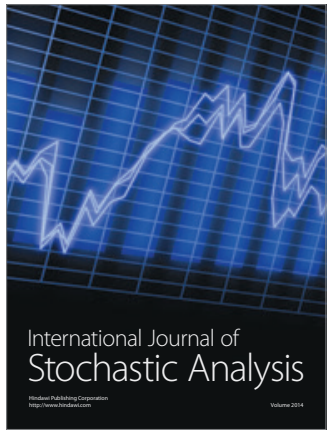

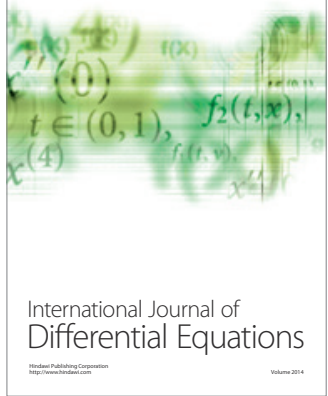
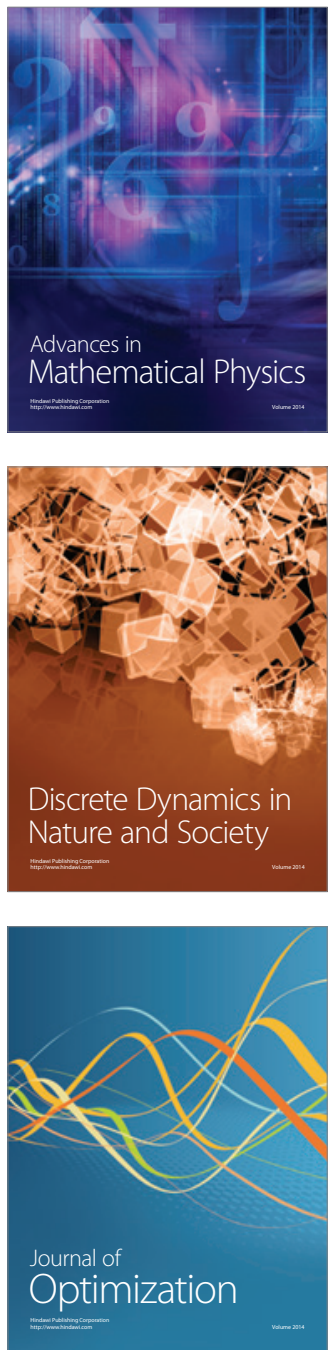